\title{
Fortalecimiento de la dirección de proyectos de una fundación en el sector educativo en Colombia, con un enfoque de oficina de proyectos, Estudio de caso
}

\section{Enhancement of project management on a foundation in educational sector in Colombia}

with a project office approach

Case study

\author{
Sandra Caicedo** \\ Leonardo Fernández \\ Édgar Ortiz \\ Wilson Sánchez
}

\footnotetext{
* Cómo citar este artículo: Caicedo, S., Fernández, L., Ortiz, É., Sánchez, W. (2019). Fortalecimiento de la dirección de proyectos de una fundación en el sector educativo en Colombia, con un enfoque de oficina de proyectos. Estudio de caso. Revista CIFE: Lecturas de Economía Social, 21(35), 61-79. DOI: https://doi.org/10.15332/22484914.5370

* Ingeniera Industrial de la Escuela Colombiana de Ingeniería, Especialista en Gerencia de Proyectos de la Universidad EAN. Estudiante de la Maestría en Gerencia de Proyectos de la Universidad EAN. Correo electrónico: scaicedo4507@universidadean.edu.co

* Ingeniero de Sistemas y Telecomunicaciones de la Universidad Sergio Arboleda. Especialista en Gerencia de Proyectos de la Universidad EAN. Estudiante de la Maestría en Gerencia de Proyectos de la Universidad EAN. Correo electrónico: jfernand9@universidadean.edu.co

Ingeniero de Sistemas de la Universidad EAN y Especialista en Gerencia de Proyectos de la misma institución. Actualmente estudia la Maestría en Gerencia de Proyectos de la Universidad EAN. Correo electrónico: eortiz_2@universidadean.edu.co

* Ingeniero Industrial de la Universidad Distrital Francisco José de Caldas, Especialista en Gerencia de Proyectos de la Universidad EAN y estudiante de la Estudiante Maestría en Gerencia de Proyectos de la Universidad EAN. Correo electrónico: wsanchez9517@universidadean.edu.co
} 


\section{Resumen}

Para el desarrollo de este estudio de caso se llevó a cabo un análisis de la gestión de proyectos en una fundación en el sector educativo en Colombia. Como resultado del análisis se pudo establecer que, siendo una empresa enfocada a desarrollar consultoría en contratación pública orientada a proyectos, la fundación carece de metodologías de gestión de proyectos claramente definidas y documentadas, repercutiendo directamente en el resultado esperado y en los procesos de seguimiento y gestión que la fundación debe realizar. Como respuesta a estas problemáticas, se plantea un fortalecimiento de la dirección de proyectos con un enfoque de oficina de proyectos que pueda proveer servicios de apoyo; todo esto alineado a la metodología del PMI, en procura de obtener una alineación de la estrategia con el portafolio de proyectos, mejorar el cumplimiento de los objetivos trazados, mejorar las competencias internas en gestión de proyectos y reforzar la cultura organizacional.

Palabras clave: oficina de gestión de proyectos, portafolio de proyectos, cultura organizacional, estrategia organizacional.

Clasificación JEL: M10, O22, M14, L19.

\section{Abstract}

For the development of this case study, an analysis of project management in a Foundation in the education sector in Colombia was carried out. As a result of the analysis, it was established that, being a company focused on developing project-oriented public contracting consultancy, the foundation lacks clearly defined and documented project management methodologies, having a direct impact on the expected result and on the monitoring and management processes that the foundation must carry out. In response to these problems, a strengthening of project management is proposed with a project office approach, which can provide support services, all aligned with the methodology of the PMI, in an attempt to obtain an alignment of the strategy with the project portfolio, improve compliance with the objectives set, improve internal skills in project management and strengthen the organizational culture.

Keywords: project management office, project portfolio, organizational culture, corporate strategy.

Classification JEL: M10, O22, M14, L19. 


\section{Introducción}

La Fundación, de la cual no es posible revelar el nombre por políticas de confidencialidad, es una entidad sin ánimo de lucro con 30 años en el sector educativo y social en Colombia y que se encuentra registrada, vigilada y controlada por la Alcaldía Mayor de Bogotá, D.C., cuenta entre 7 y 10 proyectos simultáneamente, con costos superiores a los 2000 millones cada uno, posee una planta de 250 empleados y 10 gerentes de proyecto (dependiendo de la cantidad de proyectos licitatorios). Lo que la hace una empresa de vanguardia en educación a través de consultoría con enfoque de contratación pública orientada a cumplir los pliegos de licitaciones a través de la gestión de los proyectos. Sin embargo, con base en el análisis se detectó que, por los resultados obtenidos de los proyectos de los últimos dos años, se evidencia una falta de metodología definida y documentada que permita a los directores de área y a los gerentes de proyecto la estandarización de sus procesos y procedimientos, y de este modo actuar a tiempo sobre las dificultades que se puedan presentar en su desarrollo, como reprocesos y desviaciones en tiempos de entrega, costos y calidad.

Por otro lado, la organización no tiene claramente definidos y documentados sus objetivos estratégicos, lo que impide orientar los resultados de los proyectos en este sentido; tampoco cuenta con una política para el manejo de los riesgos del portafolio de sus proyectos, estos son gestionados en función de cada cliente. Adicional a ello, no cuentan con sistemas de información que faciliten la gestión corporativa, y solo se utiliza un repositorio virtual para almacenar la información de cada proyecto de manera independiente.

Así mismo, se manifiestan dificultades para la retención del personal por el alto grado de rotación requerido para los diferentes proyectos, deficiencias en la formalización y divulgación de los procesos de la organización, así como de las condiciones laborales y el clima organizacional. En general, no se cuenta con un método, herramientas, métricas y estándares que permitan realizar una gestión de proyectos enfocada hacia el cumplimiento de los objetivos estratégicos de la Fundación, por lo que se evidencia la falta de una estructura organizacional con enfoque de oficina de proyectos que fortalezca y centralice su gestión. Por lo tanto, se plantearon las siguientes preguntas de investigación.

1 ¿Cuáles son las prácticas actuales de gestión de proyectos de la Fundación?

2 ¿Cómo debería ser estructurada la Dirección de Proyectos para fortalecer sus funciones de oficina de proyectos y que pueda responder a las necesidades de la Fundación?

3 ¿Cómo se debería implementar el plan para fortalecer la Dirección de Proyectos en la Fundación? 
Con el diagnóstico realizado y las preguntas planteadas, este caso de estudio nos permite plantear y proponer un alcance y objetivo de investigación, orientado hacia el fortalecimiento de la dirección de proyectos de la Fundación con un enfoque de oficina de proyectos.

\section{Revisión de la literatura}

Para establecer un marco de referencia teórico que sustente la propuesta, ya que la fundación hace seguimiento y control de los proyectos debido a la experiencia adquirida en los últimos 30 años en el mercado, se determinó en primer lugar definir correctamente qué es y qué hace una oficina de proyectos (Hill, 2004), cuáles serían las funciones a cumplir que ayuden a la fundación, evaluar la cultura organizacional (Ariza, 2017a) y la efectividad de los proyectos anteriores (Ariza, 2017b), para delimitar su alcance, y, por último, realizar una medición de la madurez (Kerzner, 2001, p. 156) de la Fundación en gestión de proyectos que nos permita determinar claramente la mejor solución.

\subsection{Definición de oficina de proyectos}

Existen muchos autores que dan una definición diferente de lo que es una Oficina de Proyectos, desde Hill (2004) que dice que una oficina de proyectos es una parte integradora de las organizaciones con el propósito de optimizar el uso de recursos y procesos, alinear personal y usar herramientas para la gestión del desempeño de estos. Hasta las definiciones dadas por el PMI en las diferentes ediciones de su Guía de los fundamentos, en las primeras ediciones, la definieron como

Cuerpo o entidad de la organización que tiene varias responsabilidades asignadas con relación a la dirección centralizada y coordinada de aquellos proyectos que se encuentran bajo su jurisdicción. Las responsabilidades de una oficina de gestión de proyectos pueden variar, desde realizar funciones de apoyo para la dirección de proyectos hasta ser realmente los responsables de la dirección de un proyecto. (PMI, 2008, p. 375)

Y para finalizar, en la sexta y última edición del PMBOK, que definen la oficina de proyectos como "una estructura de la organización que estandariza los procesos de gobernabilidad relacionados con el proyecto y facilita el intercambio de recursos, metodologías, herramientas y técnicas” (PMI, 2017a, p. 48), cuya definición se acopla a la propuesta para dar solución a la problemática actual de la Fundación. 


\subsection{Funciones de la Oficina de Proyectos}

Por otra parte, también se establecieron las funciones que cumpliría la oficina de proyectos a partir de Hill (2004), en la que existen 20 funciones clasificadas en 5 grupos de la siguiente manera:

- Administración de la práctica: encargada de proveer un marco de referencia para ejecutar las actividades de la Gerencia de Proyectos.

- Administración de la infraestructura: encargada de facilitar el establecimiento de un ambiente de trabajo más profesional de Gerencia de Proyectos.

- Integración de recursos: es la encargada de administrar la disponibilidad, competencia y desempeño de los recursos.

- Soporte técnico: proporciona asesoramiento y soporte a los gerentes de proyecto e integrantes del equipo en temas concernientes a Gerencia de Proyectos.

- Alineación empresarial: es la encargada de introducir a la compañía al ambiente de Gerencia de Proyectos.

En este orden de ideas, la propuesta es una oficina de proyectos como soporte técnico y con alineación empresarial para solucionar los principales problemas en la fundación.

\subsection{Tipos de oficina de proyectos}

Para determinar correctamente cuál sería el tipo de oficina de proyectos para la fundación se empleó la división de acuerdo a Hill (2004) en cuanto a su nivel de competencia y avance, descrita a continuación:

- Etapa 1: Oficina de Proyectos (Project Office). Es la unidad principal y su objetivo es lograr los resultados del proyecto en costo, cronograma y recursos. Tiene un uno o más proyectos y cuenta con un gerente de proyecto.

- Etapa 2: PMO Básica (Basic PMO). En este nivel se hace seguimiento y control a más de un proyecto y posee varios gerentes de proyectos. Establece la forma como se llevan la Gerencia de Proyectos en la organización en cuanto a herramientas comunes, procesos y prácticas

- Etapa 3: PMO Estándar (Standard PMO). Para este nivel la empresa ya considera esencial la Gerencia de Proyectos como competencia empresarial. Además del seguimiento y control, también se realiza soporte para optimizar el desempeño tanto de individuos como de proyectos en general. Adicionalmente, en este nivel la Oficina 
Revista CIFE / ISSN: 0124-3551 e-ISSN: 2248-4914 / Bogotá-Colombia / Vol. 21 N. 35 / julio-diciembre 2019 / pp. 61 -79

de Proyectos facilita la práctica profesional de los gerentes de proyecto y coordina y colabora en el manejo de interesados

- Etapa 4: PMO Avanzada (Advanced PMO). En esta etapa se encuentran las oficinas de proyectos que colaboran con las áreas de la compañía en la implementación y uso de la gerencia de proyectos en el ambiente empresarial; esta fase se encarga de integrar los intereses y objetivos empresariales a la gerencia de proyectos al introducir prácticas comunes.

- Etapa 5: Centro de Excelencia (Center of Excellence). Generalmente, se convierte en una unidad de negocio independiente dentro del compañía y se alinea con los objetivos estratégicos de la misma. Su rol principal es alinear estratégicamente la compañía, la gerencia de proyectos y su mejora continua; adicionalmente, conduce los estudios que evalúan su desempeño y funcionalidad dentro de los objetivos y como impactan el desempeño general.

Como conclusión se propuso una PMO Avanzada en etapa 4, ya que se evaluaron los recursos disponibles y se alinearon los resultados a esta propuesta.

\subsection{Cultura organizacional en el ámbito de proyectos}

Es muy importante determinar cómo la Fundación se comporta en la ejecución de los proyectos, para esto usamos el análisis de Dora Alba Ariza, Ph.D en Gerencia de proyectos y docente de la Universidad EAN, en la que define la cultura organizacional en el ámbito de los proyectos como "un marco de valores, normas y creencias colectivas compartido por los stakeholders, que se va a manifestar en actitudes y comportamientos explícitos de estos en el desarrollo de los proyectos de una organización, influenciando la efectividad de su gestión" (Ariza, 2017a, p. 232). Por tal razón, para este artículo empleamos sus conceptos para determinar la mejor solución evaluando 8 categorías culturales dentro de 4 dimensiones, como se muestra en la figura 1. 
Figura 1. Modelo de cultura organizacional en el ámbito de los proyectos

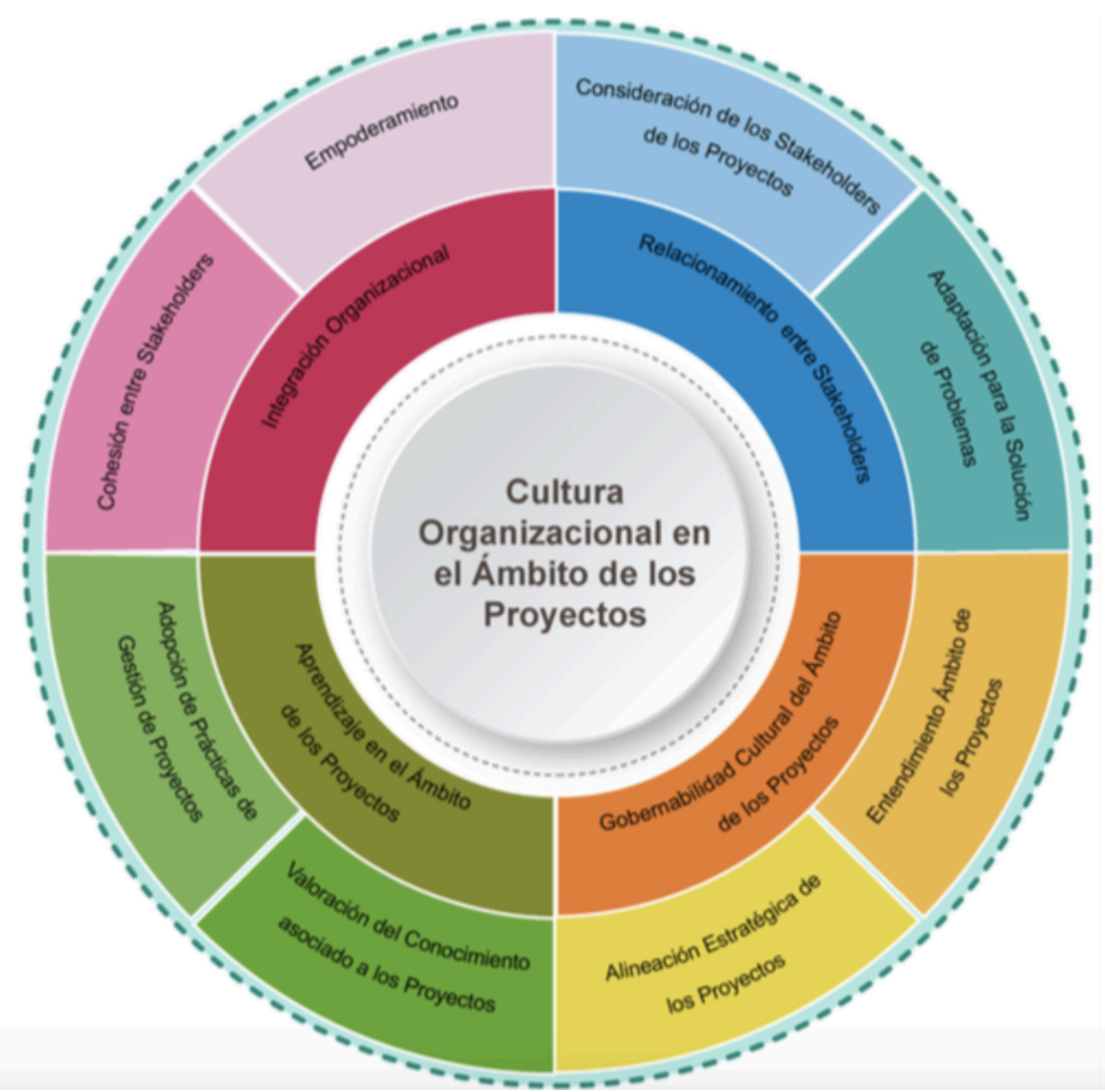

Fuente: elaboración a partir de Ariza (2017a, p. 238).

\subsection{Efectividad de la gestión de proyectos}

En cuanto a la efectividad de la gestión que dentro de esta investigación sirvió para medir qué se hizo bien en los proyectos anteriores y cómo se relaciona con la propuesta, se empleó la definición de Ariza (2017b), que dice que los criterios para medir la efectividad de los proyectos se pueden ubicar en tres categorías: 1) aspectos relacionados con la organización, 2) aspectos que se asocian a la disciplina de gestión de proyectos, y 3) aspectos que referencian resultados de los proyectos, como se muestra en la figura 2. 
Figura 2. Factores que miden la efectividad de los proyectos

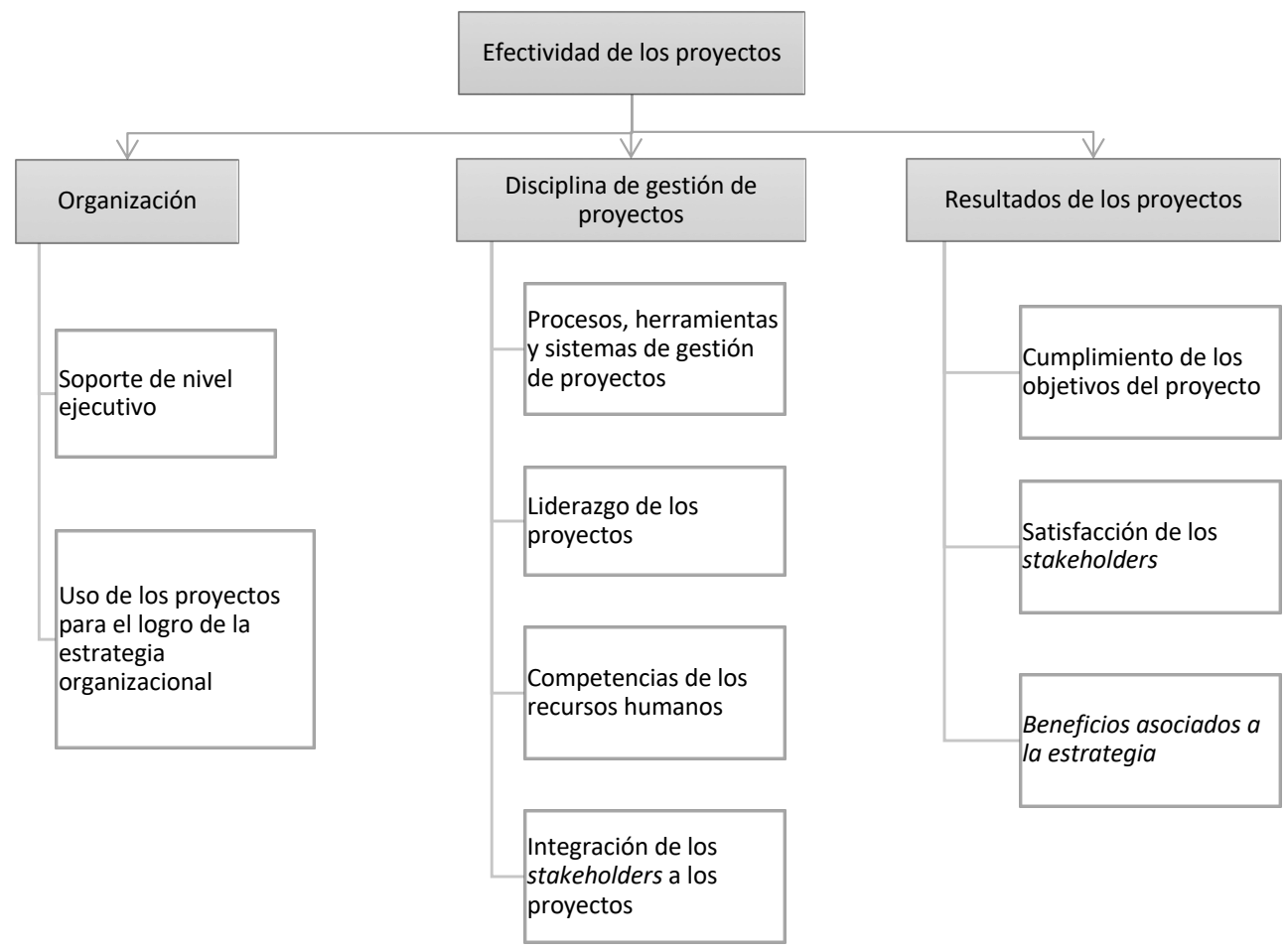

\subsection{Madurez de la gestión de proyectos}

El concepto de madurez en una organización está enfocado al reconocimiento de su desarrollo en comparación con un modelo o estándar que evoluciona progresivamente en el tiempo, siempre buscando su mejora continua. De este modo,

[...] un mayor grado de madurez significa procedimientos más efectivos en proyectos, mayor calidad en los entregables, costos más bajos, más motivación en el equipo de proyectos, una balanza deseable entre costo-cronograma-calidad, y una fundamental mejora en provecho de la organización. Por el contrario, una organización con poca madurez se caracteriza por sus repetidos errores en proyectos, aplicación de procedimientos redundantes y una historia de proyectos ejecutados sin resultados. (Levin \& Rad, 2010)

Según el PMI (2017a), un modelo de madurez es un marco de trabajo que describe las características de los procesos eficaces en áreas tan diversas como la planificación estratégica de negocios, desarrollo de negocios, ingeniería de sistemas, gestión de proyectos, gestión de riesgos, tecnología de la información o la gestión del personal.

Según Kerzner, "los modelos de madurez en administración de proyectos pueden ser utilizados para dar soporte a las empresas que realizan planeamiento estratégico y que 
buscan excelencia en su administración, los mismos permiten alcanzar madurez y excelencia en un período razonable de tiempo" (Kerzner, 2001, p. 156). Dentro de esta investigación fue de vital importancia determinar con qué grado de madurez la Fundación gestiona los proyectos para llegar a una propuesta eficiente. La figura 3 muestra los 5 niveles de madurez.

Figura 3. Los cinco niveles de madurez

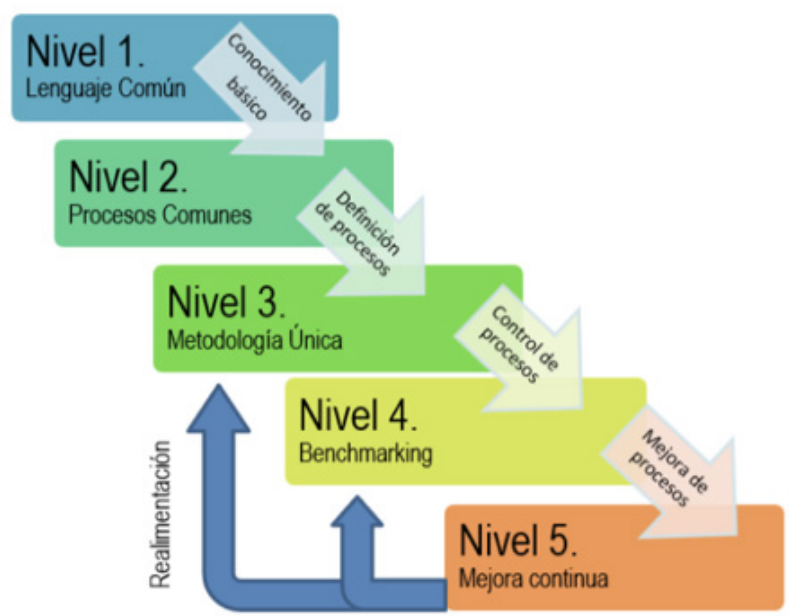

Fuente: elaboración a partir de Kerzner (2001, p. 41).

\section{Metodología de investigación y datos}

\subsection{Estudio de caso}

El objetivo del estudio de caso fue el de investigar cómo se puede fortalecer la dirección de proyectos con un enfoque de oficina de proyectos en una compañía en el sector educación en Colombia que se dedica cien por ciento a la ejecución de proyectos públicos. Para ello, se utilizaron herramientas cualitativas y cuantitativas aplicadas por medio de entrevistas estructuradas y de encuestas vía web, es decir, se trató de una investigación de tipo mixta realizada entre febrero de 2018 y abril del 2019. También se hizo un estudio correlacional, con el fin de medir el grado de relación entre algunas variables y un estudio descriptivo con el análisis e interpretación de los resultados.

Con el fin de realizar un diagnóstico completo de la Fundación, se utilizaron diferentes técnicas de recolección de datos, con el fin de cuantificar o cualificar los siguientes elementos: gestión del portafolio de proyectos, cultura organizacional en el ámbito de los proyectos, efectividad de la gestión de los proyectos, prácticas de gestión de proyectos y medición de la madurez en gerencia de proyectos. 


\subsection{Recolección de los datos}

Para determinar el estado de la Organización en cuanto a la gestión de su portafolio de proyectos, se implementó una entrevista semiestructurada, tomada de Ariza (2017a), la cual fue aplicada a tres empleados del nivel directivo de la Fundación. Estas entrevistas fueron realizadas al director de proyectos con 9 años en la Fundación, el director administrativo que tiene 10 años trabajando para la fundación y la directora de la unidad de proyectos con 16 años en este cargo, lo cual provee una visión muy real y con mucha experiencia en las encuestas. Esta herramienta se dividía en dos temas: gestión de portafolio de proyectos y cultura organizacional asociada a los proyectos.

Respecto a la cultura organizacional en el ámbito de los proyectos y efectividad de la gestión de proyectos, se realizó una encuesta de 43 preguntas, adaptada de Ariza (2017a), la cual había sido validada psicométricamente y se ajustaba al ámbito de los proyectos en Colombia. Fue implementada en una muestra probabilística de 19 personas de la Fundación de un total de 23 personas. Este instrumento permitió la realización de un análisis correlacional entre las variables dependientes (cultura y efectividad) y las variables independientes propuestas por el instrumento aplicado.

En cuanto a las prácticas de gestión de proyectos, se realizó una auditoría a tres proyectos ya ejecutados por la Fundación, utilizando un instrumento adaptado de Ariza (2017b), el cual se compone por 30 preguntas clasificadas en los 5 grupos de procesos descritos por PMI (2017a) (inicio, planeación, ejecución, monitoreo y control, y cierre).

En relación con la medición de madurez en gerencia de proyectos, se determinó que la Fundación no cuenta con procedimientos o instrumentos formales para la gestión de proyectos. Debido a esto fue necesaria la aplicación de una herramienta probada para diagnosticar la madurez de la organización. Se tomó como referencia el modelo de madurez de Kerzner (2001). Este cuestionario se encuentra agrupado en cinco niveles de Madurez (lenguaje común, procesos comunes, metodología única, benchmarking y mejoramiento continuo. Cada uno de estos grupos de niveles de madurez cuenta con una metodología diferente de medición. Este instrumento fue aplicado a tres empleados del nivel directivo de la Fundación.

\section{Resultados del estudio de caso}

Una vez aplicados los instrumentos de diagnóstico para medir la gestión del portafolio, la efectividad, las prácticas de gestión y la madurez en gerencia de proyectos de la Fundación, se realizó el registro y tabulación de la información obtenida mediante el uso de diferentes herramientas. Para el registro de la información del portafolio, las prácticas de gestión y el nivel de madurez de proyectos se configuró una base de datos 
en MS Excel, y para la efectividad de los proyectos se utilizó el paquete estadístico de análisis de datos IBM sPSs Statistics.

Según los resultados obtenidos, en la medición de la gestión del portafolio, se obtuvieron los resultados que se muestran en la tabla 1 .

Tabla 1. Resultados de la medición de la gestión del portafolio en la Fundación.

\begin{abstract}
Fortalezas
La Unidad de Proyectos Especiales de la Fundación es la encargada de la administración del portafolio de proyectos.

La alta dirección realiza un estudio de viabilidad técnica, financiera y jurídica para determinar la participación y ejecución del proyecto.
\end{abstract}

La alta dirección es quien determina la inclusión o no de nuevos proyectos al portafolio.

Los cambios al portafolio de proyectos los hace el director de proyectos, tomando como base el insumo que le entregan los gerentes de los proyectos, y lo hacen mediante reuniones de seguimiento.

Teniendo en cuenta el tipo de empresa, las metas de los proyectos son establecidas y medidas por los clientes.

Los gerentes de cada proyecto seleccionan los recursos humanos en función de los requerimientos y necesidades del cliente y del mismo proyecto.

El seguimiento a los proyectos se realiza con reuniones y comités de seguimientos en los niveles de gerencia de proyecto, dirección de proyectos, dirección de la unidad y con el director general.

Se tiene una buena comunicación con todo el personal que labora, en las reuniones de seguimiento se manifiestan las problemáticas y buscan una solución en conjunto.

Los estados de los proyectos y sus resultados son socializados con los gerentes de proyecto, director de proyectos y estos a la vez con sus equipos de trabajo.

Se identifican y conocen los beneficios que genera cada proyecto, tanto a nivel de servicio como a nivel financiero, sin embargo, no se documentan.

\section{Debilidades}

No se encuentra definido un proceso formal para la administración del portafolio.

Se realizan procesos para la identificación de riesgos en los proyectos externos, sin embargo, los mismos no son documentados en cada proyecto ni en un repositorio centralizado.

La organización no tiene una política para el manejo de los riesgos, los gestiona en función de cada cliente y proyecto.

Los riesgos los gestionan en función del presupuesto y de los entregables de cada proyecto, no tienen la visión general del portafolio.

No se han establecido criterios para la cancelación de proyectos (sanciones, multas).

No existe dentro de la compañía un patrocinador definido, dado que los proyectos corresponden al sector público y en ese escenario es cada cliente el patrocinador.

No se tienen herramientas para la gestión de los recursos, establecen contratación por prestación de servicios, o laboral a término fijo por los tiempos de duración de cada proyecto, el control lo lleva el gerente del proyecto.

No se cuenta con personal directo para labores operativas de la empresa diferente a los cargos directivos, la mayor parte del personal está asignado por proyectos y algunos son transversales.

No se cuenta con un software para la centralización de la información, se utiliza un repositorio virtual para almacenar la información de cada proyecto de manera independiente.

No se tienen claramente definidos los objetivos estratégicos de la compañía, por lo tanto, no pueden orientar los resultados de los proyectos en este sentido. 


\section{Fortalezas}

Se identifican los beneficios intangibles de los proyectos y los promulgan, dado que sirven como enganche y experiencia para la adjudicación de nuevos proyectos.

\section{Debilidades}

Se tienen problemas para la retención del personal por el alto grado de rotación requerido para los diferentes proyectos; existen deficiencias de formalización y divulgación de los procesos de la organización; se requiere realizar la planeación estratégica, mejorar las condiciones laborales y el clima organizacional.

Se busca dar cumplimiento a los requerimientos y necesidades del cliente, entregando valor a las entidades en donde se desarrollan los proyectos.

Fuente: elaboración propia.

Figura 4. Valores promedio de las variables de cultura

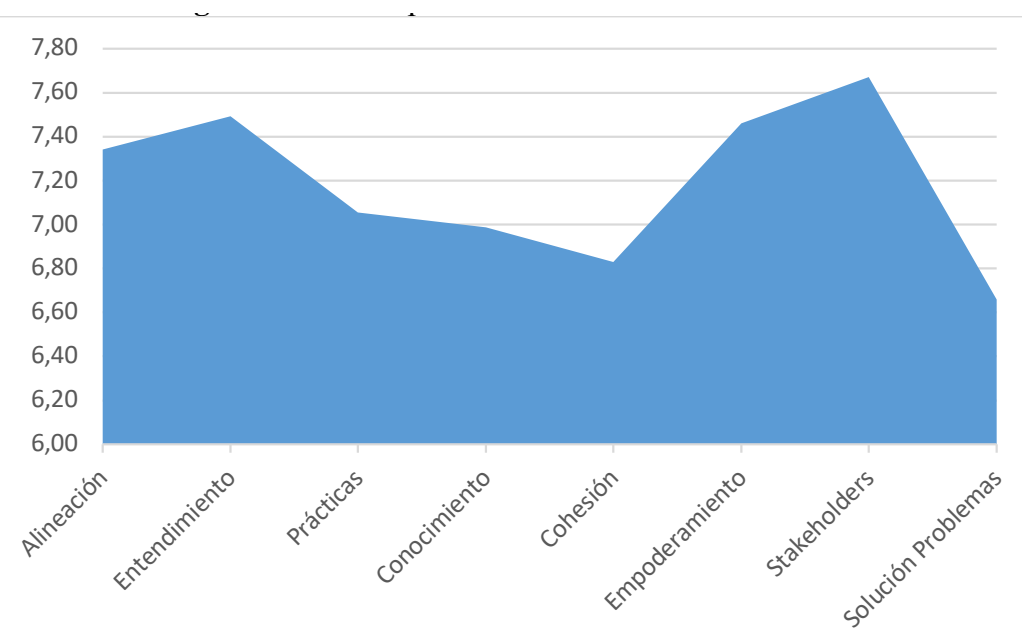

Fuente: elaboración propia.

Como se muestra en la figura 4, de acuerdo con los puntajes obtenidos en la evaluación de cultura organizacional en el ámbito de proyectos, la percepción de los encuestados indica que las categorías culturales de empoderamiento y adaptación para la solución de problemas son las características con mayor calificación, mientras las menos calificadas son las de consideración de los stakeholders, valoración del conocimiento asociado a los proyectos y cohesión entre los stakeholders. Estos resultados indican que hay oportunidades en la organización para mejorar el nivel en que se tienen en cuenta las necesidades y posición de los diferentes stakeholders en los proyectos, la valoración del conocimiento que se obtiene con su ejecución y la visión compartida de los proyectos entre las áreas de la organización, de modo que colaboren más eficazmente en aras de obtener los objetivos de los proyectos. 
La efectividad en la gestión de proyectos, que se obtuvo mediante la evaluación de 11 dimensiones, muestran los resultados que se indican en la figuras 5.

Figura 5. Valores promedio de las variables de efectividad

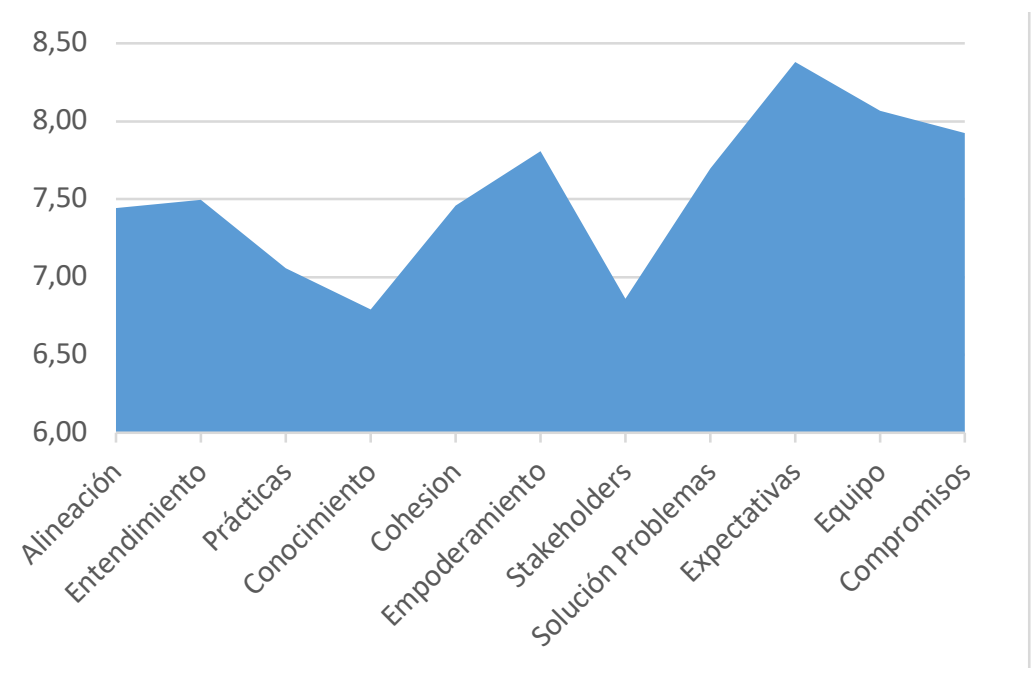

Fuente: elaboración propia.

Con los resultados obtenidos se puede deducir que la dimensión de cumplimiento de expectativas es la mejor valorada, mientras la dimensión de cumplimiento de compromisos es la de más baja calificación. Esta última incluye los indicadores de cumplimiento con cronograma, presupuesto y adecuación de las respuestas dadas a los riesgos que se presentan. De estas tres, la menor calificada es expectativas.

En cuanto al resultado en la evaluación de las prácticas de gestión de proyectos, que se evaluaron a través de los grupos de procesos y del ciclo de vida de los proyectos según el PMBOK (PMI, 2017), se obtuvieron los resultados que se muestran en la figura 6. 
Revista CIFE / ISSN: 0124-3551 e-ISSN: 2248-4914 / Bogotá-Colombia / Vol. 21 N. 35 / julio-diciembre 2019 / pp. $61-79$

Figura 6. Gráfica de resultados en prácticas de gestión de proyectos

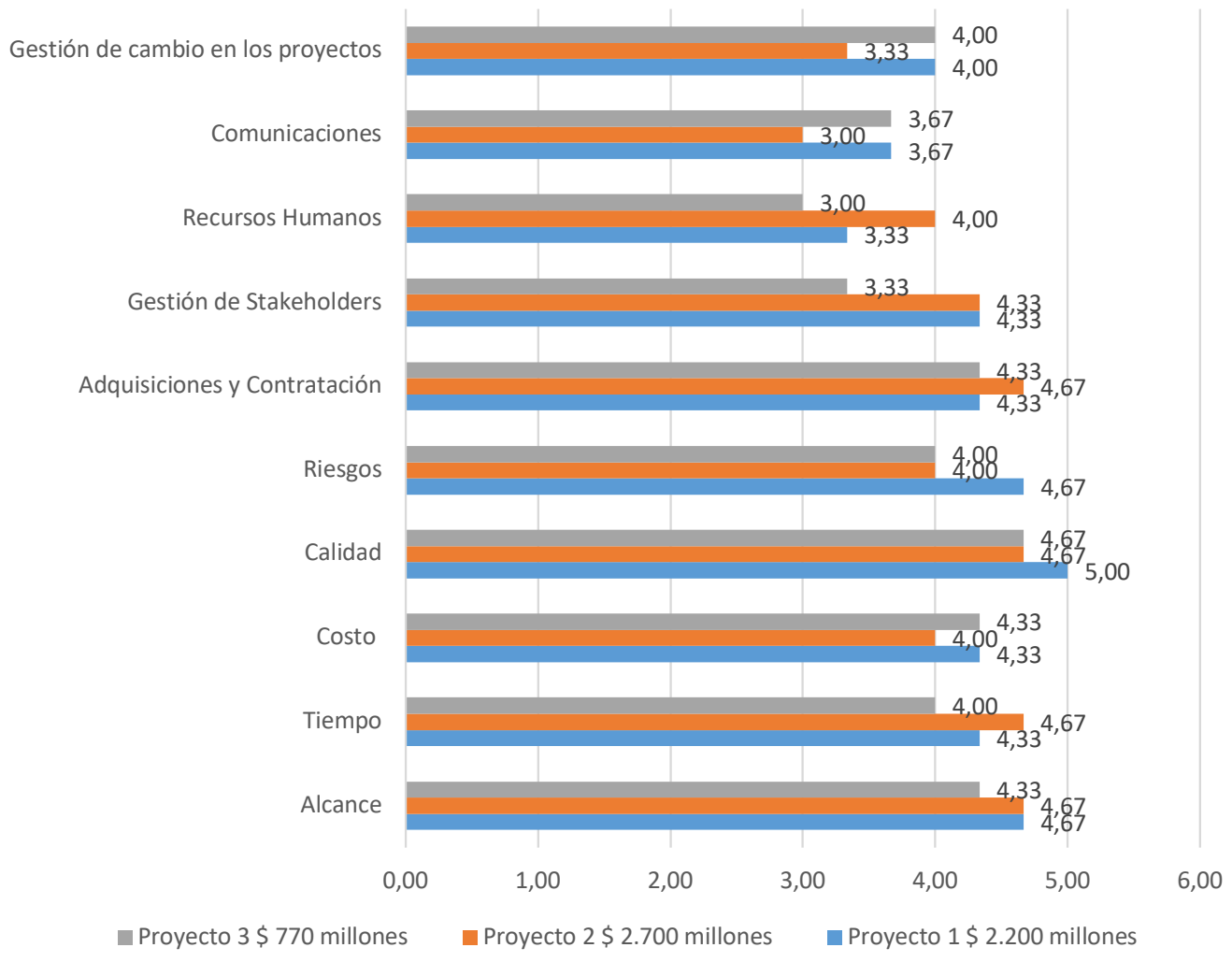

Fuente: elaboración propia.

De los resultados obtenidos en los tres proyectos seleccionados, los más altos puntajes obtenidos están en las áreas de calidad y alcance, los cual están acorde a los objetivos de la Fundación al cumplir a cabalidad las metas esperadas en los pliegos de peticiones de las licitaciones y llevar un control de las métricas a lo largo de los proyectos. Por otra parte, se encuentra una calificación menor en los recursos humanos, hecho que evidencia la falta de asignación de roles y responsabilidades dentro de los proyectos y que influencia directamente el ambiente para el desarrollo del trabajo en la fundación y, por último, se detecta la falta de seguimiento al desempeño de los equipos de trabajo con su respectiva retroalimentación.

Adicionalmente, otra baja calificación la tienen las comunicaciones en los tres proyectos, donde se identifica una falla en la incorporación de lecciones aprendidas en la planificación y análisis de riegos, importante para no cometer los mismos errores en futuros proyectos. De la misma manera, el puntaje bajo esta en la definición de los requerimientos de información y cómo estos son entregados a los stakeholders afectando la relación con el cliente, parte importante de las licitaciones. 
En la figura 7 se muestran los resultados de la encuesta separada por cada proceso (PMI, 2017). En ella se evidencia claramente una baja calificación en la planeación de los proyectos, con una puntuación de 3.8 sobre 5, en especial en la calificación de la reserva de los presupuestos y en la identificación de los riesgos asociados a estos. Por último, y no por eso el menos importante, se identifica en la auditoría que no hay una manera de aceptar o negar los cambios en el proyecto. Por otra parte, se puede observar una alta calificación en monitoreo y control, con un promedio de 4.29 sobre 5, lo que significa que en la fundación hay un énfasis en dar cumplimiento a los requerimientos del cliente, en este caso, dar seguimiento al pliego de peticiones de las licitaciones.

Figura 7. Resultados en prácticas de gestión de proyectos por cada proceso

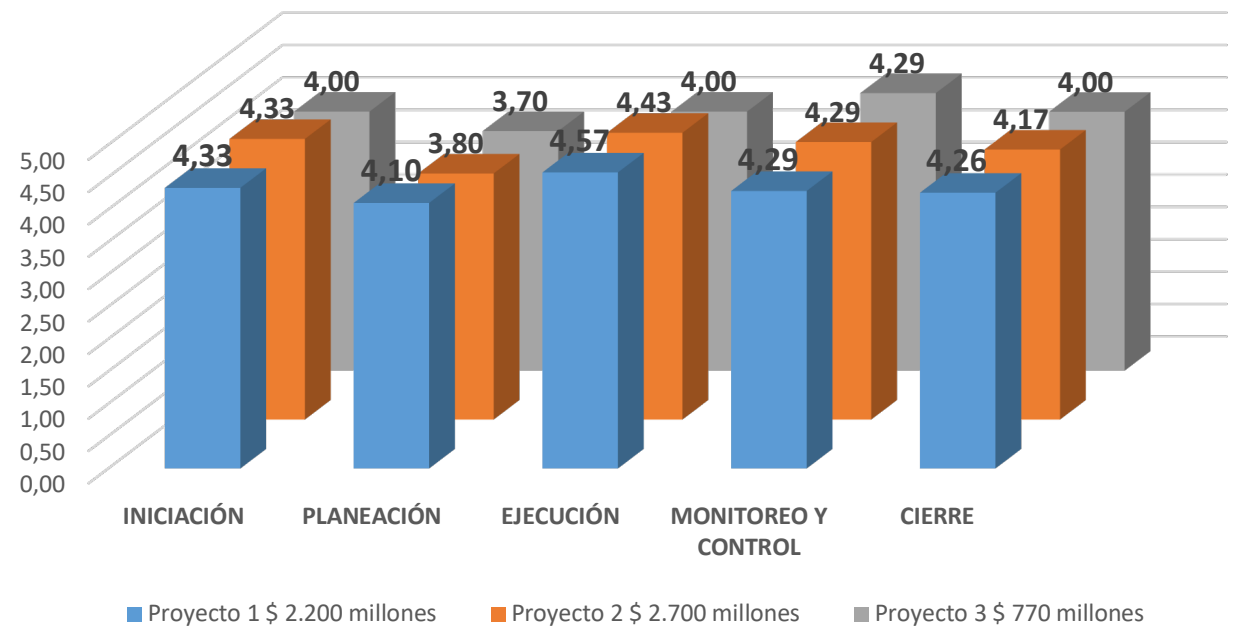

Fuente: elaboración propia.

La madurez en gerencia de proyectos se evaluó mediante 5 niveles, los cuales se presentan a continuación a través de los resultados que se muestran en la figura 8.

Figura 8. Gráfica de resultados medición de madurez en gerencia de proyectos en la fundación

\section{Lenguaje común}

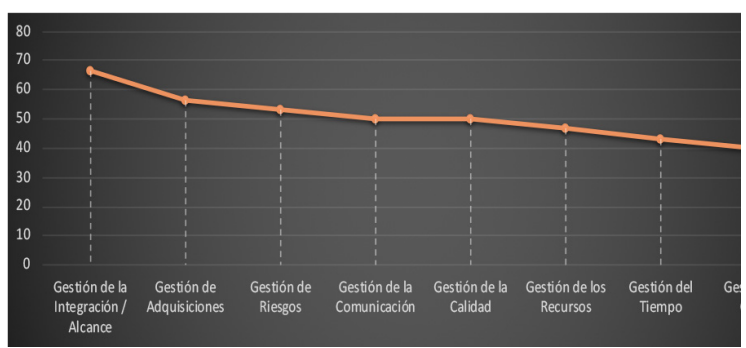


Revista CIFE / ISSN: 0124-3551 e-ISSN: 2248-4914 / Bogotá-Colombia / Vol. 21 N.o 35 / julio-diciembre 2019 / pp. $61-79$ Procesos comunes

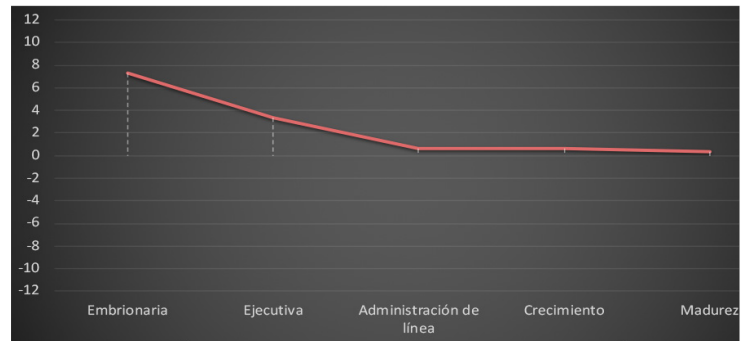

Metodología única

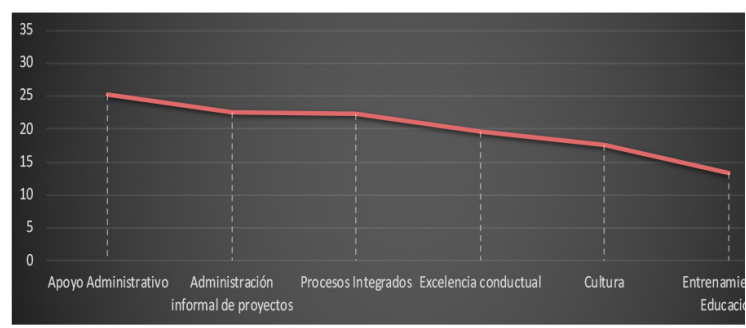

Benchmarking

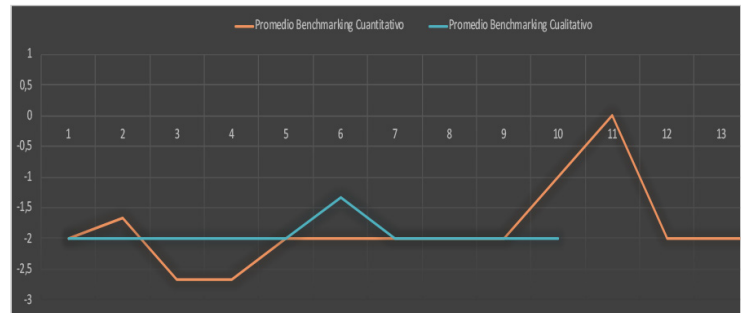

Mejoramiento continuo

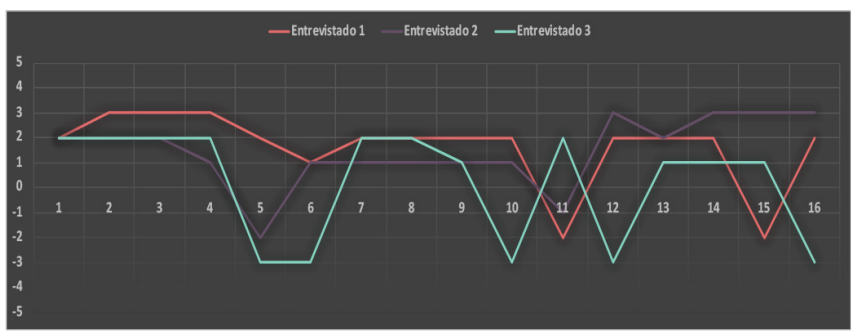

Fuente: elaboración propia.

Resultados nivel 1. Lenguaje común: la gestión de la integración/alcance es la única área que obtuvo un puntaje superior a 60; las demás áreas, en su orden gestión de adquisiciones, gestión de riesgos, gestión de la comunicación, gestión de la calidad, gestión de los recursos, gestión del tiempo y gestión del costo obtuvieron un puntaje menor a 60 
puntos, lo que significa que en estas áreas existe una deficiencia de conceptos básicos en la administración de proyectos.

Resultados nivel 2. Procesos comunes: la Fundación se encuentra en una fase embrionaria, es decir, se reconoce que la administración de proyectos puede ser beneficiosa para el cumplimiento de sus metas. Asimismo, se encuentra en proceso hacia la fase ejecutiva, lo que significa que la Fundación evoluciona hacia la aceptación de que la administración de proyectos puede ser necesaria para la sobrevivencia de la organización. Por el contrario, la Fundación aún no ha evolucionado en las fases de administración en línea, crecimiento y madurez.

Resultados nivel 3. Metodología única: la puntuación es inferior a 69 en los 6 segmentos, lo que significa que la Fundación requiere avanzar hacia la comprensión y el reconocimiento de la necesidad de integrar la totalidad de sus procesos de administración de proyectos en una metodología específica

Resultados nivel 4. Benchmarking: la puntuación es inferior a 5 tanto en la evaluación cuantitativa como cualitativa, lo que significa que a la Fundación le falta adquirir un compromiso con el establecimiento de una marca de referencia o contra quién compararse. Debe enfocarse en la investigación y mejoras de la metodología y los procesos, así como en la forma en que la cultura ejecuta esa metodología

Resultados nivel 5. Mejora continua: la puntuación es inferior a 9, lo que indica que existe una fuerte resistencia al cambio en la Fundación y que falta soporte de gestión superior para la mejora continua. Es importante analizar la importancia de medir los resultados obtenidos en el proceso de benchmarking, de manera que logren mejorar su metodología.

Para concluir, se puede decir que la fundación está en un nivel inicial de madurez (nivel 1 del modelo de madurez de Kerzner), reconociendo que se tienen implementados algunos elementos del componente de gestión de proyectos, con existencia de avances informales. A pesar de que se han realizado ejercicios para analizar la información de proyectos para hacer seguimiento y control a alcance, tiempo y costo, estos no se realizan de una manera estructurada, estandarizada y continua.

\section{Conclusiones}

En el presente artículo se analizaron las prácticas actuales de gestión de proyectos en una fundación en el sector educativo en Colombia. Para este análisis, se inició con la investigación de literatura especializada en la temática de gestión de proyectos a nivel organizacional, luego se aplicaron cuatro instrumentos de diagnóstico: el primero para 
medir la gestión del portafolio de proyectos, el segundo para medir la efectividad de los proyectos a partir de aspectos clave de su cultura organizacional, el tercero para evaluar las prácticas de gestión de los proyectos en una muestra de tres proyectos, y el cuarto para medir la madurez en gerencia de proyectos de la Fundación.

Los resultados de la investigación muestran deficiencias en la gestión del portafolio de proyectos, particularmente en su alineación con los objetivos estratégicos y la definición de un proceso formal para su administración. En cuanto a la efectividad de los proyectos, se encontraron oportunidades de mejora en la gestión de las expectativas de los stakeholders, la valoración del conocimiento obtenido a través de la ejecución de los proyectos y una visión compartida entre las áreas de la Fundación. En referencia a las prácticas de gestión de proyectos, existen debilidades en el cumplimiento de cronograma, presupuesto y adecuación de las respuestas dadas a los riesgos que se presentan. En cuanto a madurez en gerencia de proyectos, la Fundación se encuentra en una etapa inicial, donde se reconoce la importancia y la necesidad de un buen entendimiento, metodología y lenguaje para gestionar sus proyectos de manera estructurada, estandarizada y continua.

En referencia a la estructuración, se identificó que el enfoque de la Dirección de Proyectos debe ser no solo de control sino de soporte, donde sus principales funciones estén encaminadas al acompañamiento y asesoría a los gerentes de proyecto en la estandarización de la gestión de proyectos y la articulación entre las partes interesadas; así mismo, implementar los controles y métricas necesarias para asegurar el cumplimiento de los entregables de los proyectos con criterio de calidad y eficiencia.

Finalmente, para fortalecer la Dirección de Proyectos se recomienda iniciar la implementación con planes de capacitación para los equipos de los proyectos, buscando el mejoramiento de su conocimiento en lenguaje y procesos comunes en la gestión de proyectos. Del mismo modo, se recomienda implementar un proceso de gestión de cambio enfocado a sustituir la cultura informal por una estructura formal que permita realizar predicciones en tiempos y movimientos, acciones, disponibilidad y uso de recursos, seguimiento a hitos, ruta crítica, planes de contingencia, gestión de riesgos, entre otras.

Se recomienda a futuros investigadores con proyectos similares que se profundice sobre la aplicación de herramientas tecnológicas que permitan estandarizar las prácticas de gestión entre proyectos del mismo tipo, potencializando las lecciones aprendidas y optimizando la curva de aprendizaje de los equipos de trabajo.

\section{Agradecimientos}

Agradecemos a la Fundación por permitirnos desarrollar este trabajo de investigación y análisis dentro de su compañía, a las directivas, gerentes de proyectos y personal en 
general que participaron de las encuestas y entrevistas realizadas. A la Universidad EAN, por permitirnos estos espacios investigativos para fortalecer nuestra base de conocimiento y ampliar la del país en cuanto a gestión de proyectos.

\section{Referencias bibliográficas}

Ariza, D. A. (2017a). Construcción de un modelo de cultura organizacional en el ámbito de los proyectos y su aplicación en el sector de tecnología de la información en Colombia (tesis de doctorado). Universidad EAN.

Ariza, D. A. (2017b). Efectividad de la gestión de los proyectos: una perspectiva constructivista. Obras y Proyectos, 22, 75-85. https://doi.org/10.4067/ S0718-28132017000200075

Hill, G. M. (2004). Evolving the Project Management Office: A Competency Continuum. Information Systems Management, 21(4), 45-51. https://doi.org/10.1201/1078/44705.2 1.4.20040901/84187.6

Kerzner, H. (2001). Strategic planning for project management using a project management maturity model. John Wiley \& Sons, Inc,.

Levin, G., \& Rad, P. (2010). The Advanced Project Management Office. In The Advanced Project Management Office. https://doi.org/10.1201/9781420000375

Project Management Institute. (2008a). Guía de los fundamentos para la dirección de proyectos (Guía del PMBOK). Guarta ed., Project Management Institute. Ed. Newtown Square, Pennsylvania: Project Management Institute, Inc.

Project Management Institute. (2017a). Guía de los fundamentos para la dirección de proyectos (Guía del PMBOK). Sexta ed., Project Management Institute. Ed. Newtown Square, Pennsylvania: Project Management Institute, Inc.

Project Management Institute. (2017b). The Standard for Portfolio Management. 4th ed., Pensylvania: Project Management Institute, Inc. 